\title{
An Innovated 80V-100V High-Side Side-Isolated N-LDMOS Device
}

\author{
Shao Ming Yangi ${ }^{1,3^{*}}$, Gene Sheu ${ }^{1}$, Ting Yao Chien ${ }^{2}$, Chieh Chih $\mathrm{Wu}^{2}$, Tzu Chieh $\mathrm{Lee}^{2}$, Ching Yuan $\mathrm{Wu}^{1}$, Chiu Chung Lai ${ }^{2}$ \\ ${ }^{1}$ Department of Computer Science and Information Engineering, Asia University, 500, Lioufeng Rd., Taichung 41354, Taiwan. \\ ${ }^{2}$ School of Software and Microelectronics, Peking University, Beijing, China. \\ ${ }^{3}$ Department of Medical Research, China Medical University Hospital, China Medical University, Taichung, Taiwan.
}

\begin{abstract}
We used TCAD Synopsys 3D tools and device simulators to propose an innovative device structure of $80 \mathrm{~V}-100 \mathrm{~V}$ high-side NLDMOS by using the silicon to silicon-di-oxide ratio with side trench. The high-side can also be developed by placing an NBL structure which can deliver a high as over 200V isolation voltage. The $3 \mathrm{D}$ structure can clear see the optimized linear $\mathrm{p}$-top and n-drift region have better charge balance with linear doping profile to get the benchmark breakdown voltage (BVdss) of 80V with onresistance (Ron) as low as $130 \mathrm{~m} \Omega-\mathrm{mm}^{2}$ and $100 \mathrm{~V}$ with on-resistance as low as $175 \mathrm{~m} \Omega-\mathrm{mm}^{2}$. The linear p-type buried layer using high dosage and lower energy to achieve the better SOA and higher isolation voltage. Optimized linear p-top and PBL can improve Ron by $32.5 \%$ compare to other $100 \mathrm{~V}$ high side device which have done from reference.
\end{abstract}

\section{Introduction}

For $50 \mathrm{~V}-140 \mathrm{~V}$ smart power device is highly demanded by auto and motor driver's industry. In order to get high performance for ESOA and TSOA [1], some study use expensive technology for process and complication of structure that increase cost, such as silicon-on-insulator (SOI), double epitaxial layer [2], use n-well and p-well together etc. Kirk effect show NPN of $\beta$ issue that is serious for on-state I-V curve and affect ESOA performance [3].

Comparison to low-side, high-side need a best junction isolation breakdown and also sustain the lateral breakdown which use NBL ( $\mathrm{n}+$ buried layer) between the source and the substrate. In this work, we propose a new innovated [4] high-side side-isolated n-channel LDMOS 3D device on both front and back edges of the extended drift region with thickness epitaxial layer. A double-RESURF concept incorporates an additional layer (linear p-top layer) [5] inside the n-drift extended region to get the charge balance, the net doping will clear see the linear profile curve, it can improve on-state resistance and keep high breakdown, the good charge balance not only get lower Ron and high breakdown also reduce the device length to cost down.

To avoid the transistor beta, cause the Kirk effect, we use linear p-type buried layer (PBL) [6] on top of n-type layer (NBL) inside the epitaxy layer, the balance between PBL and NBL need to consider the isolation BV can't be destroy, so the linear PBL give the small transistor beta and also make the good profile curve to sustain the high isolation breakdown. The device is $3 \mathrm{D}$ simulation. Each region is optimized by several experiments. The process was optimized using a simulation written in TCAD Synopsys 3D tools to determine.

\section{Device structure and conditions}

This NLDMOS High Side device in this study is using $0.35 \mu \mathrm{m}$ process with shallow N-epitaxial drift region, simulated with TCAD Synopsys tools and device simulators.

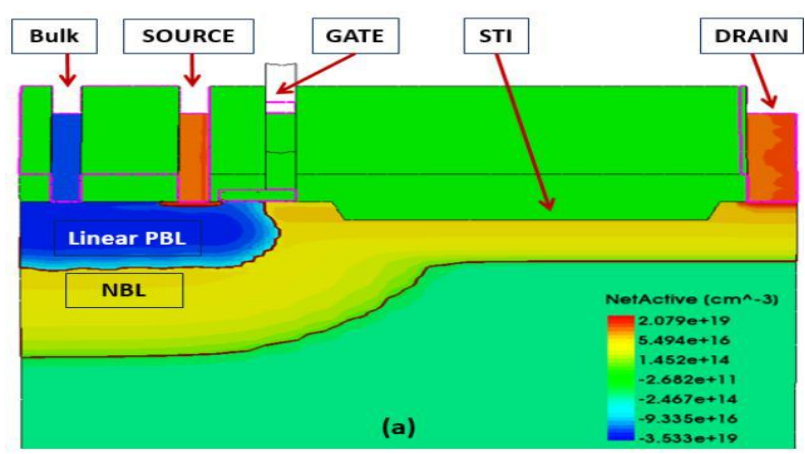

Figure 1 shows our novel device cross section structure, and simulated top view in Synopsys with device length $7.6 \mu \mathrm{m}$. Table 1 show this work simple condition. For this novel device, channel length of $0.35 \mathrm{um}$ is formed by p-body diffusion doped at $3 \times$ $10^{17} \mathrm{~cm}-3$, drift region is doped at $4.65 \times 10^{16} \mathrm{~cm}-3$, and source and drain contacts are all doped at $1 \times 10^{19} \mathrm{~cm}^{-3}$.

'Corresponding author: rickyyang121@asia.edu.tw 
The gate oxide composed of $\mathrm{SiO}_{2}$ is $120 \mathrm{~A}$ thick with a gate length of $0.75 \mu \mathrm{m}$.

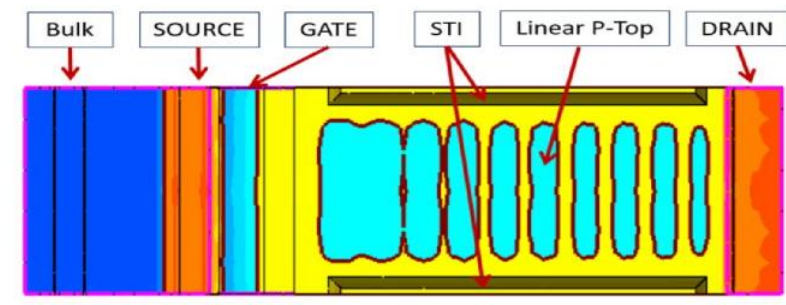

(b)
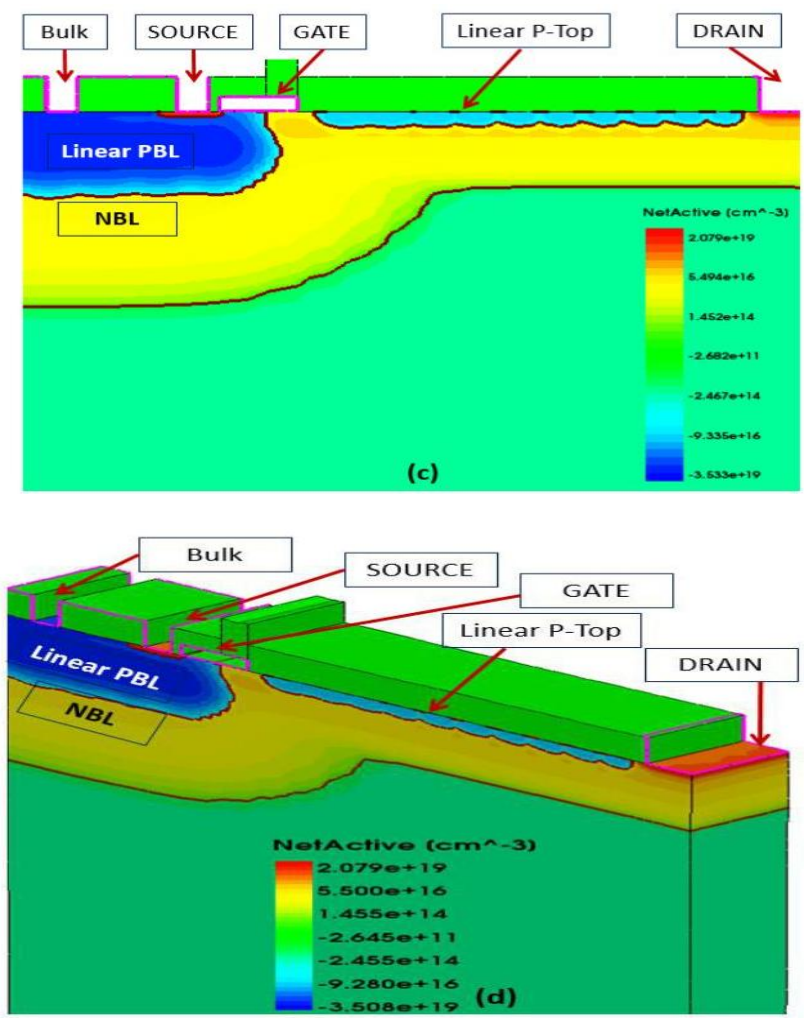

Fig. 1. Schematic of 3D (a) High-Side Side-Isolated NLDMOS proposed structure; (b) top view; (c) front view; (d) rotation view.

Table 2. The parameters which using in the TCAD Synopsys 3D simulation.

\begin{tabular}{|c|c|c|}
\hline Parameters & Unit & Values \\
\hline Substrate & ohm-cm & 100 \\
\hline N-epi & ohm-cm & 0.145 \\
\hline Epi thickness & um & 0.6 \\
\hline p-body & $\mathrm{cm}-2$ & $3 \mathrm{e}^{13} \sim 3 \mathrm{e}^{14}$ \\
\hline n-type buried layer & $\mathrm{cm}-2$ & $5 \mathrm{e}^{12}$ \\
\hline n-type blanket & $\mathrm{cm}-2$ & $7 \mathrm{e}^{11} \sim 9 \mathrm{e}^{11}$ \\
\hline Source contact & $\mathrm{cm}-3$ & $1 \mathrm{e}^{19}$ \\
\hline Drain contact & $\mathrm{cm}-3$ & $1 \mathrm{e}^{19}$ \\
\hline
\end{tabular}

The key technique that implemented in this work are linear highly doped with lower energy of p-type buried layer and linear p-top region. The linear highly doped of p-type buried layer using high dosage and lower energy to get the charge balance in the deep n-type buried layer, the doping profile showed in figure 2 , the linear curve can provide high isolation breakdown also make the better SOA.

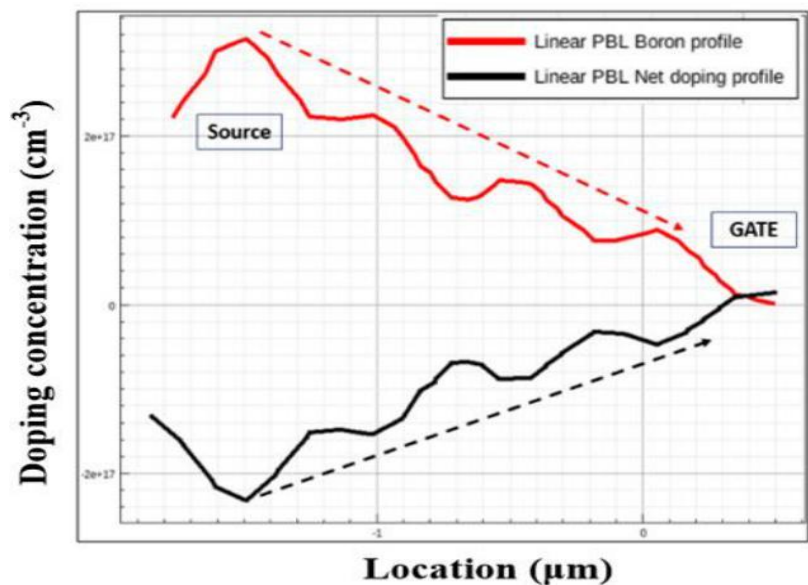

Fig. 2. Linear p-type buried layer and n-type buried layer doping profile, the curve present not only in boron profile also net doping profile have good linear trend.

The linear p-top made the good charge balance and present the linear doping profile in the n-drift region showed in figure 3 , the linear trend curve redistribution the surface charge to make the high breakdown also improve $\mathrm{R}_{\mathrm{ON}}$.

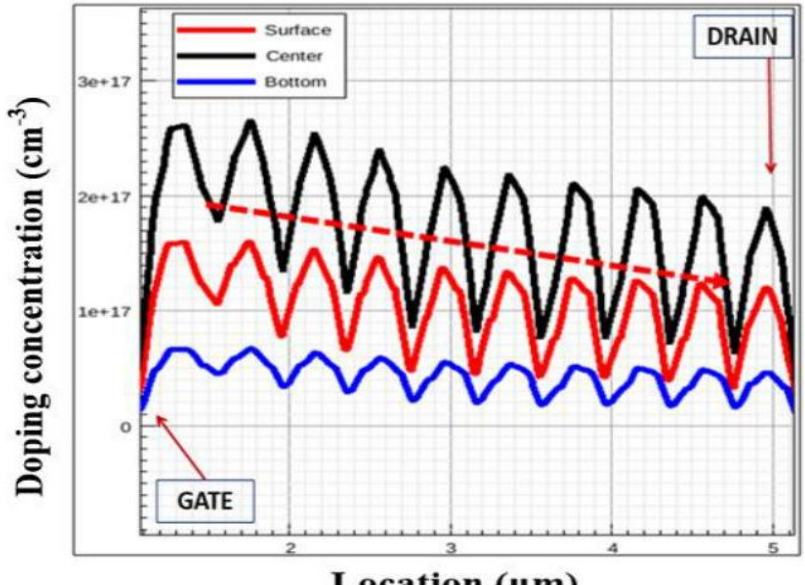

Fig. 3. Linear p-top region has good charge balance in N-drift region, the profile in different location all have linear trend curve.

\section{Result and discussions}

\subsection{Device breakdown and SOA}

From double RESURF concept, we put the p-top of shallow region in the $n$-drift region surface in our novel device. P-top region have a linear trend curve relationship with the $\mathrm{n}$-drift region for charge balance. This linear trend curve not only get the high lateral breakdown but also have benchmark on-resistance.

For the High Side structure, we placed the N-buried layer under the source side. The shallow n-drift region cannot make a good sensitivity for P-buried layer and $\mathrm{N}$ - 
buried layer. In order to maintain a good isolation breakdown and reduce the beta of the parasitic NPN transistor, we innovate the linear $\mathrm{p}$-buried layer. There by, the device has low leakage current and avoid on-state curves of Kirk effect. It not only can improvement the SOA curve but also have the good isolation breakdown as we can see in Figure 4.

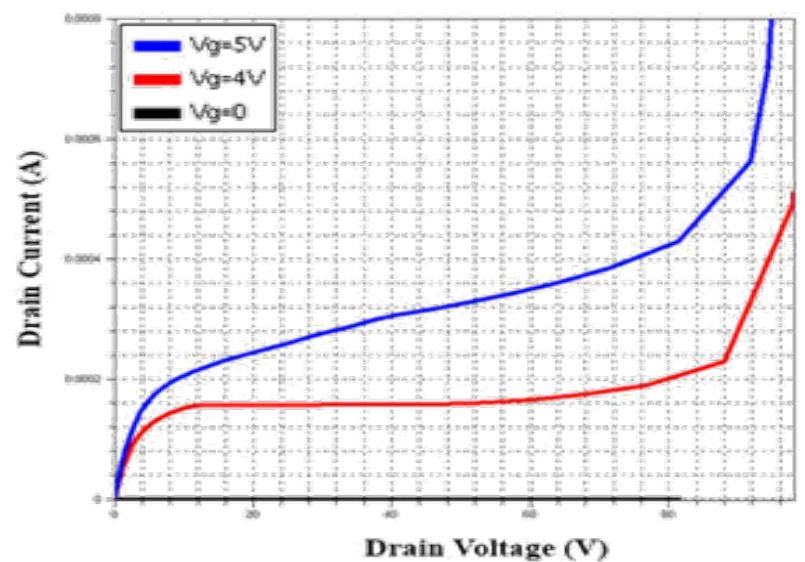

(a)

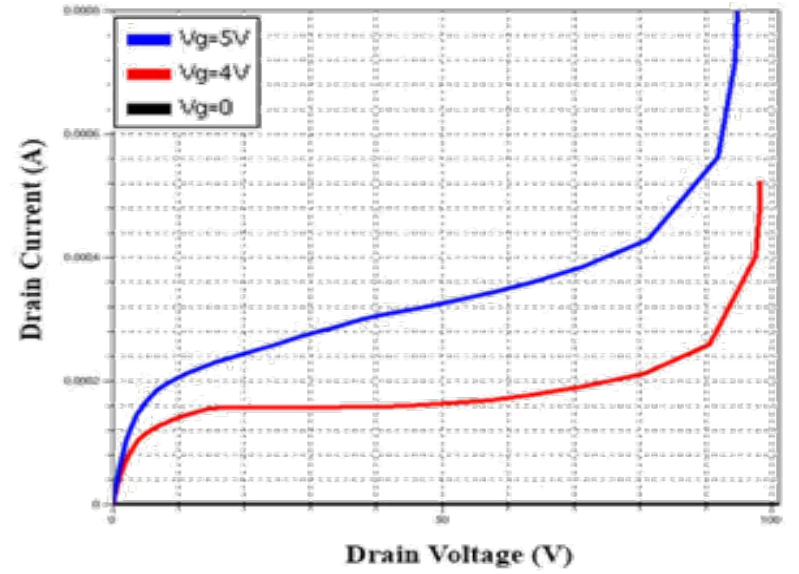

(b)

Fig. 5. Id vs. Vd characteristics of device for (a) $80 \mathrm{~V}$ and (b) 100V High-Side Side-Isolated NLDMOS of off-state breakdown voltage at $\mathrm{Vg}=0$ and on-state breakdown voltage at $\mathrm{Vg}=4 \mathrm{~V}$ and $\mathrm{Vg}=5 \mathrm{~V}$ in $3 \mathrm{D}$ simulation

\subsection{Optimized characteristics of device}

In order to consider the cost and charge balance, this paper of $80 \mathrm{~V}-100 \mathrm{~V}$ High-Side Side-Isolated NLDMOS device use the same size of device length, width and height. To analysis simulation of result, figure 6 shows that adjustment phosphorus dosage of n-blanket for our innovated 80V-100V High-Side Side-Isolated NLDMOS device design.

The curve of relationship can observe more phosphorus dosage of n-blanket that lateral breakdown voltage and Ron decreasing in direct proportion; otherwise increasing. In this work, for our $80 \mathrm{~V}$ HighSide Side-Isolated NLDMOS use 9e11 of n-blanket dosage can get $81.8 \mathrm{~V}$ of lateral breakdown voltage and Ron is $112 \mathrm{~m} \Omega * \mathrm{~mm}^{2}$. For $100 \mathrm{~V}$ voltage use $7 \mathrm{e} 11$ can get $101 \mathrm{~V}$ of lateral breakdown voltage and Ron is $118 \mathrm{~m}$ $\Omega * \mathrm{~mm}^{2}$.

Figure 7 shows the result of breakdown voltage and on-resistance compare with some of the conventional high-side NLDMOS and this work has the best Ron for $80 \mathrm{~V}-100 \mathrm{~V}$.

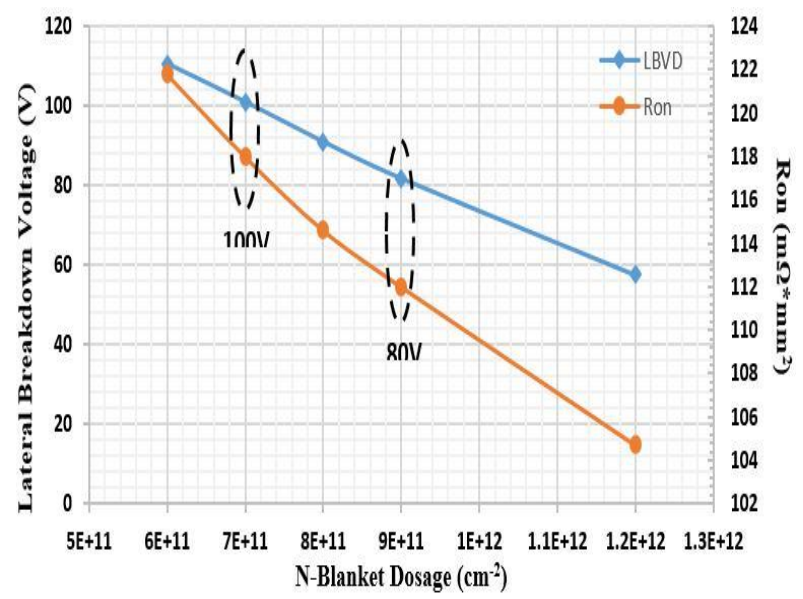

Fig. 6. Both of the off-state breakdown voltage and onresistance curves as a different $\mathrm{N}$-blanket dosage for innovation $80 \mathrm{~V}-100 \mathrm{~V}$ High-Side Side-Isolation NLDMOS device.

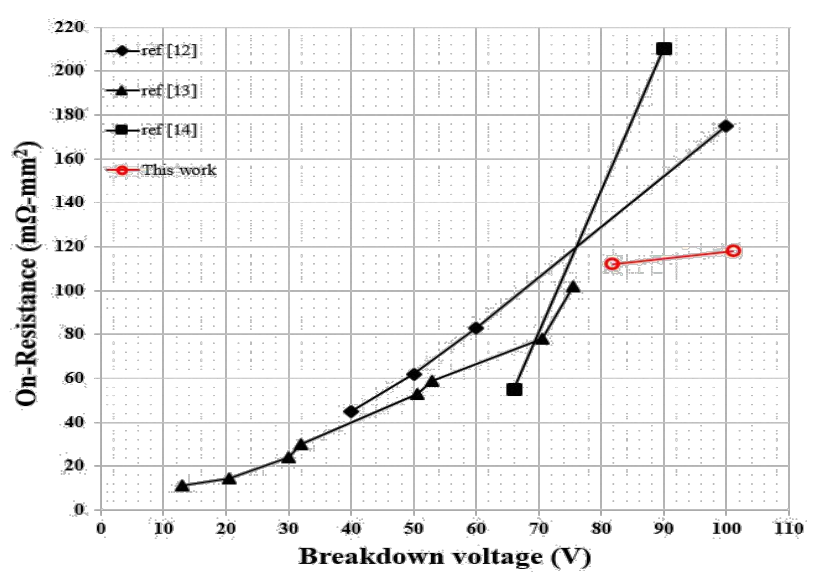

Fig. 7. Trade-off characteristics between the breakdown voltage and the specific on-resistance.

\section{Conclusion}

The $80 \mathrm{~V}-100 \mathrm{~V}$ High-Side NLDMOS with SidedIsolated structure still produce high Ron due to the drift length limit. The newly invented device structure using short drift length with sided isolation can deliver high lateral breakdown and high isolation breakdown as well with low Ron. We implemented NBL ( $\mathrm{n}+$ buried layer) which to be located beneath the source region to prevent punch-through breakdown between the source and the p-substrate, also get high isolation breakdown without affecting other characteristics. In addition, the device with added linear p-type layer and linear p-top can reduce Kirk effect and get high breakdown with better low Ron.

Acknowledgment: The authors would also like to thank National Center for High-performance computing, National Nano Device Laboratories, and the National Chip Implementation Center for supporting us. 


\section{References}

1. P. Hower and S. Pendharkar, "Short and Long-Term Safe Operation Area Considerations in LDMOS Transistors", IEEE, (2005) pp 545-550.

2. P. Holland and P Igic, "A p+/p-buffer/n-epi cmos compatible high-side RESURF LDMOS transistor for Power IC applications", Microelectronics Journal 38, 762-766 (2007)

3. R. Zhu, V. Parthasarathy, V. Khemka, A. Bose and T. Roggenbauer, "Implementation of High-Side, High-Voltage RESURF LDMOS in a sub-half Micron Smart Power Technology", IEEE, (2001)

4. J.A. Appels and H.M.J. Vaes, "High Voltage Thin Layer Devices (RESURF Devices)", IEEE, (1979)

5. S. Hardikar, M. M. De Souza, Y.Z. Xu, T.J. Pease and E.M. Sankara Narayanan, "A Novel Double RESURF LDMOS for HVIC's", Microelectronics Journal 35, Issue 3, 305-310 (2004)

6. P. Hower, J. Lin, S. Pendharkar, B. Hu, J. Arch, J. Smith and T. Efland, "A Rugged LDMOS for LBC5 Technology", in Proc. 17th Int. Symp. Power Semiconductor Devices \& IC's, May 23-26, Santa Barbara, CA (ISPSD 2005), pp. 327-330 